\title{
Antimicrobial Activity of Traditional Chinese Medicines on Common Oral Bacteria
}

\author{
Michelle K. Z. Yuen ${ }^{1}$, Ricky W. K. Wong ${ }^{1}$, Urban Hägg ${ }^{1}$, Lakshman Samaranayake ${ }^{2}$ \\ ${ }^{1}$ Orthodontics, Faculty of Dentistry, The University of Hong Kong, Hong Kong, China \\ ${ }^{2}$ Oral Biosciences, Faculty of Dentistry, The University of Hong Kong, Hong Kong, China \\ E-mail: fyoung@hkucc.hku.hk \\ Received January 24, 2011; revised March 8, 2011; accepted March 15, 2011
}

\begin{abstract}
Objective: To evaluate twenty Traditional Chinese Medicines (TCM) against four oral bacteria. Methods: Twenty TCM were tested for sensitivity against Streptococcus mitis, Streptococcus sanguis, Streptococcus mutans and Porphyromonas gingivalis. Aliquots of suspension of each bacterial species were inoculated on a horse blood agar (HBA) plate, $6 \mathrm{~mm}$ diameter paper disks was soaked in different drug suspensions were placed concentrically on a HBA plate. Disks soaked in $0.2 \% \mathrm{w} / \mathrm{v}$ chlorhexidine were used as positive controls. These HBA plates were incubated for 48 hours anaerobically and the diameters of growth inhibition of three different areas were measured using a calibrated computer software and the mean diameter obtained for each bacteria. Broth microdilution assay was used to determine minimum inhibitory concentration (MIC) and minimum bactericidal concentration (MBC). The experiment was repeated on three separate occasions. Results: The TCMs that consistently against Porphyromonas gingivalis, included Folium artemisiae argyi, Fructus crataegi, Rhizoma dryopteris crassirhizomae, Flos magnoliae, Rhizoma polygoni cuspidati, Radix scrophulariae ningpoensis, Galla chinensis, Radix scutellariae baicalensis and Rhizoma coptidis; against Streptococcus mutans included Fructus crataegi, Galla chinensis and Rhizoma copitidis; against Streptococcus mitis and Streptococcus sanguis included Galla chinensis and Rhizoma copitidis. Conclusion: Rhizoma copitidis and Galla chinensis had inhibitory effects on Streptococcus mitis, Streptococcus sanguis, Streptococcus mutans and Porphyromonas gingivalis in vitro.
\end{abstract}

Keywords: Chinese Medicine, Antimicrobial Activity, Streptococcus mutans, Streptococcus sanguis, Streptococcus mitis, Porphyromonas gingivalis, Oral Biofilm

\section{Introduction}

Dental caries is a common human disease that affects a vast majority of people. It is a chronic endogenous infection caused by the normal oral commensal flora [1]. Oral biofilm develop on all natural or artificial shedding and non-shedding surfaces of the oral cavity. Microorganisms in oral biofilm are the major aetiological agents of dental caries. Other than caries, oral biofilm can cause many oral infections including periodontal disease and candidiasis. Plaque formation involves initial colonization and multiplication by pioneer species, followed by secondary colonization by other species and finally becoming a climax community [2]. The "pioneer species" of oral biofilm are Streptococcus oralis, Streptococcus mitis and Streptococcus sanguis. There are also specific bacteria that are closely related to specific dental diseases, for example, Stre ptococcus mutans and Porphyromonas gingivalis are associated with dental caries and periodontal disease, respectively [1].

Traditional Chinese Medicines (TCM) has been used in China to treat various infectious diseases for more than four thousand years. Different from the westernmedicine, TCM works as a formula of herbs that is tailored to individual patient under their specific condition.

They are designed in the form of remedy that uses one or two main ingredients that target the illness, with many other ingredients also added to adjust the formula to suit patient's condition [3]. Recently the mechanism of one of these formulae has been investigated at molecular, cellular and organism levels [4]. TCM possess a variety of biological properties that has the potential to be de- 
veloped as effective drugs. Certain TCM have been shown to have antibacterial properties and so far none has shown to have any known resistance. Currently, a number of TCMs has already been used in oral healthcare products such as toothpaste according to their effects. Yet few studies have been performed to screen these TCM and evaluate their effectiveness against oral bacteria forming oral biofilm.

The twenty TCMs are selected according to their antibacterial properties that enable them to treat infection and disease at different part of body. The aim of this study was to evaluate in vitro twenty TCMs that are currently used to treat infectious diseases; for their antimicrobial activity against oral biofilm bacteria and caries and periodontal disease. The hypothesis is to investigate the TCMs investigated in the study have effect against the four common oral bacteria.

\section{Methods}

\subsection{Organism and Culture Condition}

Frozen isolates of type cultures of Streptococcus mitis (ATCC 15914), Streptococcus sanguis (ATCC 10556), Streptococcus mutans (ATCC 35668) and Porphyromonas gingivalis (ATCC 33277) were thawed and their identity reconfirmed using standard methodology. They were then inoculated onto horse blood agar (HBA) and incubated anaerobically at $37^{\circ} \mathrm{C}$ for 3 days. For sensitivity studies the bacterial cultures were suspended in phosphate buffered saline at a concentration of $1 \times 10^{6}$ cells/mL (0.5 MacFarland Standard Units).

\subsection{Identification and Preparation of TCM Extracts}

The twenty TCM were purchased from a local Chinese Medicine store and were identified morphologically, histologically and chemically using standard Chinese herbal identification procedures [5].

Aqueous extracts of TCM were prepared using standard protocol [5]. Briefly, $4 \mathrm{~mL}$ distilled water was added to $10 \mathrm{~g}$ of TCM powder. The mixture was boiled with constant stirring for 4 hours with occasional adding of distilled water to prevent drying. Distilled water was added at the end to make up the volume of the mixture to $4 \mathrm{~mL}$. The mixture was cooled, centrifuged and filtered. This produced $2.5 \mathrm{~g} / \mathrm{mL}$ of one TCM extract.

The twenty TCM chosen for the study were Rhizoma coptidis, Radix arnebia, Herba artemisiae, Flos magnoliae, Radix bupleuri, Galla chinensis, Folium artemisiae argyi, Radix scrophulariae ningpoensis, Radix scutellariae baicalensis, Rhizoma polygoni cuspidati, Folium isatidis, Fructus crataegi, Herba patriniae cum radice, Rhizoma dryopteris crassirhizomae, Spica prunellae vulgaris, Radix sophorae, Fructus gardeniae jasminoidis, Anemarrhena aspodeloidea Bunge, Cortex fraxini and Tarxacum mongolicum. Chlorhexidine gluconate, a common oral antiseptic, at a concentration of $0.2 \% \mathrm{w} / \mathrm{v}$ was used as a positive control for all experiments.

\subsection{Agar Diffusion Assay}

The standard agar diffusion assay for sensitivity testing was performed according to a standard protocol [1]. 20 $\mu \mathrm{L}$ aliquots of suspension of each bacterial species were inoculated on horse blood agar (HBA) plates using glass rods, then $6 \mathrm{~mm}$ diameter paper disks soaked in $10 \mu \mathrm{L}$ of each of the $2.5 \mathrm{~g} / \mathrm{mL}$ TCM extract were placed concentrically on the HBA plate. Positive controls were disks soaked in $10 \mu \mathrm{L}$ of $0.2 \% \mathrm{w} / \mathrm{v}$ chlorhexidine placed in the HBA plates. These HBA plates were incubated anaerobically for 48 hours at $37^{\circ} \mathrm{C}$. After that, measurements of any growth inhibition zone were evaluated using a calibrated computer software (Image J 1.40 for Windows, NIH Image, Maryland, USA). The diameters of growth inhibition of three different directions were measured and the mean diameter of growth inhibition was calculated for each organism. The experiment was repeated on three separate occasions. In cases where no clear bacteria growth inhibition could be seen but the bacteria surface appearance changed, partial inhibitory effect was recorded.

\subsection{Broth Microdilution Susceptibility Test}

The TCMs, Rhizoma coptidis and Galla chinensis, which showed potent antimicrobial activity against the four tested bacteria in the agar diffusion assay-screening test, were selected for minimum inhibitory concentration (MIC) determination using the standard broth microdilution assay [6]. Inocula of 24 hours bacteria cultures were standardized to a turbidity equivalent of 0.5 McFarland standard at $520 \mathrm{~nm}$ with a spectrophotometer. The suspensions were further diluted in Rosewell Park Memorial Institute (RPMI) 1640 medium (Life technologies, New York, USA) to yield an inoculum concentration of ap-

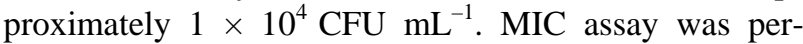
formed in 96-well round-bottomed microtiter plates (Iwaki, Tokyo, Japan) and each of the bacteria was exposed to a double dilution of each of TCM agents. The plates were covered with a lid and incubated for 72 hours at $37^{\circ} \mathrm{C}$ in an anaerobic chamber to evaluate the growth kinetics. The MIC of each TCM drug was defined as the lowest concentration that prevents visible turbidity of the broth. Same procedure had also been carried out on a 
horseblood agar plate to evaluate the minimal bactericidal concentration (MBC) which classified as the lowest concentration of drug that kills at least $99.9 \%$ of the CFUs contained in the original inoculums. Experiments were repeated on three different occasions with duplicate determinations on each occasion.

\subsection{Statistic Analysis}

Data were analysed with a statistical analysis computer software (SPSS 15.0 for Windows $\complement$, Chicago, USA). Data were performed with one-way ANOVA to compare the effects of different TCMs. Differences of pair-wise comparison of each TCM with the positive control were considered significant when the $\mathrm{p}$ value was less than 0.05. All TCMs that were showed effective against individual bacteria in this experiment proved to be significant except when Folium artemisiae argyi was tested against $P$. gingivalis (Table 1).

\section{Results}

\subsection{Initial Biofilm forming Bacteria (Strepto- coccus mitis, Streptococcus sanguis)}

The mean of inhibition zones of chlorhexidine measured were $7.4 \mathrm{~mm}$ against Streptococcus mitis compared to Galla chinensis and Rhizoma copitidis, which were of $5.8 \mathrm{~mm}$ and $6.2 \mathrm{~mm}$ respectively (Table 1, Figures 1 and 2). The average mean of inhibition zone measured in diameter of chlorhexidine was $9.1 \mathrm{~mm}$ against Streptococcus sanguis, where as Galla chinensis and Rhizoma copitidis were measured as $10.6 \mathrm{~mm}$ and $7.6 \mathrm{~mm}$. Rhizoma copitidis demonstrated a comparable effect to chlorhexidine against Streptococcus mitis, while Galla chinensis showed sign of stronger effect than the chlorhexidine (9.1 mm) against Streptococcus sanguis.

\subsection{Caries Causing Bacteria (Streptococcus mutans)}

The mean of inhibition zones of chlorhexidine measured were $12.1 \mathrm{~mm}$ against Streptococcus mutans. Three out of twenty TCM extracts tested demonstrated consistent antimicrobial activities with zones of growth inhibition ranging from $5 \mathrm{~mm}$ to $12 \mathrm{~mm}$ against Streptococcus mutans. The average mean of inhibition zones measured in diameter of Fructus crataegi, Galla chinensis and Rhizoma copitidis to Streptococcus mutans, are $5.4 \mathrm{~mm}, 9.6$ $\mathrm{mm}$ and $11.4 \mathrm{~mm}$ respectively (Table 1, Figure 3). When compared to the positive control, chlorhexidine, which is a highly potent antibacterial agent, Rhizoma copitidis demonstrated a similar effect to chlorhexidine (12.1 mm).
Table 1. Table showing antimicrobial property of TCMs.

\begin{tabular}{|c|c|c|c|c|}
\hline & S. mutans & S. mitis & $\begin{array}{c}\text { S. } \\
\text { sanguis }\end{array}$ & $\begin{array}{c}P . \\
\text { gingivalis }\end{array}$ \\
\hline Cortex fraxini & - & - & - & $\mathrm{P}$ \\
\hline Flos magnoliae & - & - & - & $\begin{array}{c}9.6 \\
( \pm 0.1)^{*}\end{array}$ \\
\hline $\begin{array}{l}\text { Folium artemisiae } \\
\text { argyi }\end{array}$ & - & - & - & $\begin{array}{c}7.2 \\
( \pm 0.6)\end{array}$ \\
\hline Folium isatidis & - & - & - & $\mathrm{P}$ \\
\hline Fructus crataegi & $\begin{array}{c}5.4 \\
( \pm 0.3)^{*}\end{array}$ & - & $\mathrm{P}$ & $\begin{array}{c}7.3 \\
( \pm 0.1)^{*}\end{array}$ \\
\hline $\begin{array}{l}\text { Fructus gardeniae } \\
\text { jasminoidis }\end{array}$ & - & - & - & - \\
\hline Galla chinensis & $\begin{array}{c}9.6 \\
( \pm 0.1)^{*}\end{array}$ & $\begin{array}{c}5.8 \\
( \pm 0.2)^{*}\end{array}$ & $\begin{array}{c}0.6 \\
( \pm 0)^{*}\end{array}$ & $\begin{array}{c}20.2 \\
( \pm 0.2)^{*}\end{array}$ \\
\hline Herba artemisiae & - & - & - & $\mathrm{P}$ \\
\hline $\begin{array}{l}\text { Herba patriniae } \\
\text { cum radice }\end{array}$ & - & - & - & - \\
\hline $\begin{array}{l}\text { Herba taraxaci } \\
\text { mongolici cum } \\
\text { radice }\end{array}$ & - & - & $\mathrm{P}$ & $\mathrm{P}$ \\
\hline Radix arnebia & - & - & - & $\mathrm{P}$ \\
\hline Radix bupleuri & - & - & - & - \\
\hline $\begin{array}{l}\text { Radix scrophulariae } \\
\text { ningpoensis }\end{array}$ & - & - & $\mathrm{P}$ & $\begin{array}{c}19.4 \\
( \pm 0.1)^{*}\end{array}$ \\
\hline $\begin{array}{l}\text { Radix scutellariae } \\
\text { baicalensis }\end{array}$ & - & - & $\mathrm{P}$ & $\begin{array}{c}25.3 \\
( \pm 0.1)^{*}\end{array}$ \\
\hline Radix sophorae & - & - & $\mathrm{P}$ & - \\
\hline Rhizoma anemarrhenae & - & - & - & - \\
\hline Rhizoma coptidis & $\begin{array}{l}11.4 \\
( \pm 0)^{*}\end{array}$ & $\begin{array}{c}6.2 \\
( \pm 0.2)^{*}\end{array}$ & $\begin{array}{c}7.6 \\
( \pm 0)^{*}\end{array}$ & $\begin{array}{c}56.3 \\
( \pm 0.2)^{*}\end{array}$ \\
\hline $\begin{array}{l}\text { Rhizoma dryopteris } \\
\text { crassirhizomae }\end{array}$ & - & - & - & $\begin{array}{c}9.2 \\
( \pm 0.3)^{*}\end{array}$ \\
\hline $\begin{array}{l}\text { Rhizoma polygoni } \\
\text { cuspidati }\end{array}$ & - & - & - & $\begin{array}{c}12.1 \\
( \pm 0.1)^{*}\end{array}$ \\
\hline Spica prunellae vulgaris & - & - & - & $\mathrm{P}$ \\
\hline Control & $\begin{array}{c}12.1 \\
( \pm 0)^{*}\end{array}$ & $\begin{array}{c}7.4 \\
( \pm 0.1)^{*} \\
\end{array}$ & $\begin{array}{c}9.1 \\
( \pm 0)^{*}\end{array}$ & $\begin{array}{c}23.7 \\
( \pm 0.1)^{*} \\
\end{array}$ \\
\hline
\end{tabular}

*"P” stands for partial inhibitory effect; *“_” indicates for no inhibitory effect; *Values in parenthesis indicate standard deviation; *“*” indicate significant differences $(\mathrm{p}<0.05)$

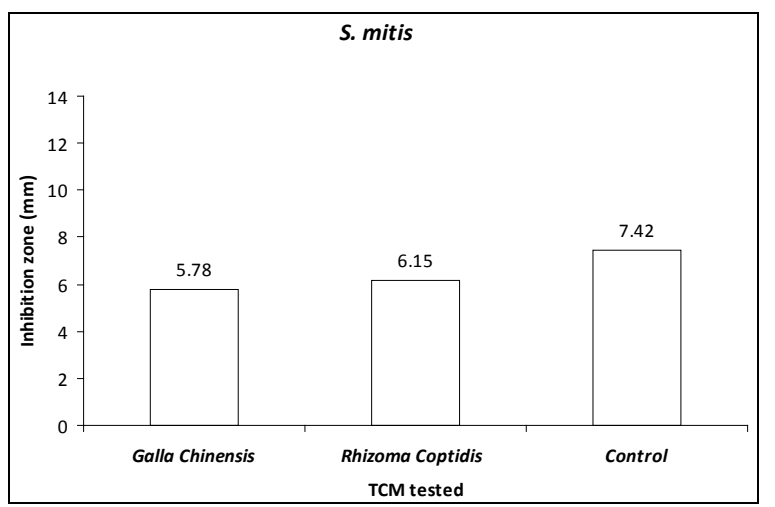

Figure 1. Sensitivity of $S$. mitis to 2 out of 20 TCM tested shown in mean value. 


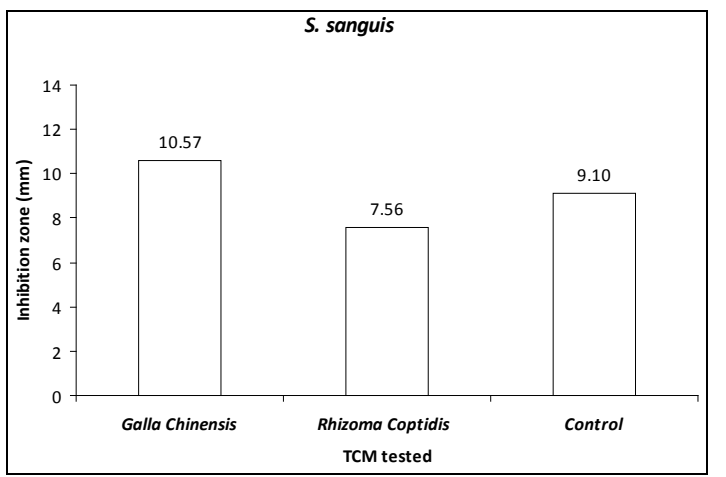

Figure 2. Sensitivity of $S$. sanguis to 2 out of TCM tested shown in mean value.

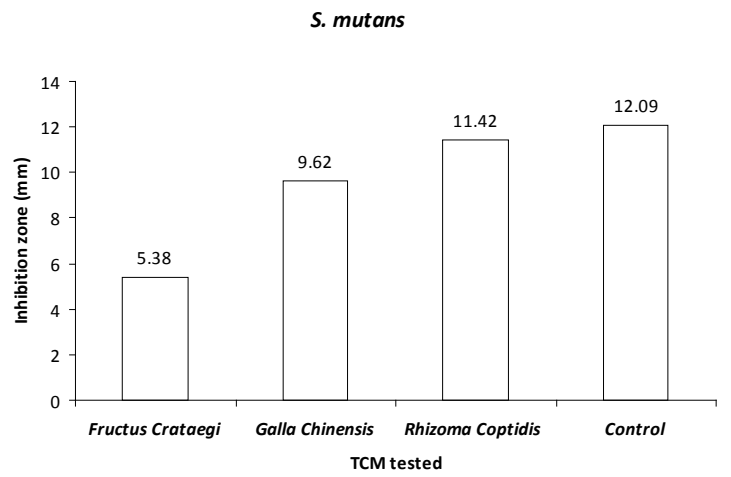

Figure 3. Sensitivity of $S$. mutans to 3 out of 20 TCM tested shown in mean value.

\subsection{Periodontal Disease Causing Bacteria (Porphyromonas Gingivalis)}

Chlorhexidine was measured to have $23.7 \mathrm{~mm}$ in average mean against Porphyromonas gingivalis. Nine of the twenty TCM extracts also demonstrated consistent antimicrobial activity with zones of growth inhibition ranging from $7 \mathrm{~mm}$ to $57 \mathrm{~mm}$ against Porphyromonas gingivalis. The sequences of drug in ascending orders of effectiveness towards Porphyromonas gingivalis were Folium artemisiae argyi $(7.2 \mathrm{~mm})$, Fructus crataegi $(7.3 \mathrm{~mm})$, Rhizoma dryopteris crassirhizomae (9.2 mm), Flos magnoliae (9.6 $\mathrm{mm})$, Rhizoma polygoni cuspidati (12.1 mm), Radix scrophulariae ningpoensis (19.4 mm), Galla chinensis (20.2 $\mathrm{mm})$, Radix scutellariae baicalensis $(25.3 \mathrm{~mm})$ and Rhizoma coptidis (56.3 mm). Galla chinensis $(20.2 \mathrm{~mm})$ and Radix scutellariae baicalensis $(25.3 \mathrm{~mm})$ demonstrated comparable effective to chlorhexidine $(28.7 \mathrm{~mm})$, while Rhizoma Coptidis shows a significant antimicrobial effect compares with chlorhexidine against Porphyromonas gingivalis (Table 1, Figure 4).

\subsection{Partial Effects}

For the remaining TCMs tested, Fructus crataegi, Radix scutellariae baicalensis, Radix scrophulariae ningpoensis, Radix sophorae and Herba taraxaci mongolici cum radice showed weak antimicrobial effects against Streptococcus sanguis. While Cortex fraxini, Herba artemisiae. Folium isatidis, Spica prunellae vulgaris, Radix arnebia and Herba taraxaci mongolici cum radice demonstrated weak antimicrobial effects against Porphyromonas gingivalis.

\subsection{Minimum Inhibitory Concentrations}

The MIC and MBC values for Rhizoma Coptidis against S. mutans were $0.039 \mathrm{~g} / \mathrm{mL}$ and $0.156 \mathrm{~g} / \mathrm{mL}$ respectively. It stated as $0.020 \mathrm{~g} / \mathrm{mL}$ and $0.156 \mathrm{~g} / \mathrm{mL}$ while against $\mathrm{S}$. mitis. And $0.039 \mathrm{~g} / \mathrm{mL}$ for both MIC and MBC values when against $S$. sanguis (Tables $\mathbf{2}$ and $\mathbf{3}$ ).

Galla chinensis when hold against $S$. mutans had the MIC value as $0.0391 \mathrm{~g} / \mathrm{mL}$ and $0.078 \mathrm{~g} / \mathrm{mL}$ as MBC value. For $S$. mitis, the MIC value was $0.010 \mathrm{~g} / \mathrm{mL}$ and MBC value was $0.078 \mathrm{~g} / \mathrm{mL}$. Both MIC and MBC values were the same when Galla chinensis against $S$. sanguis, which is $0.039 \mathrm{~g} / \mathrm{mL}$ (Tables 2 and 3).

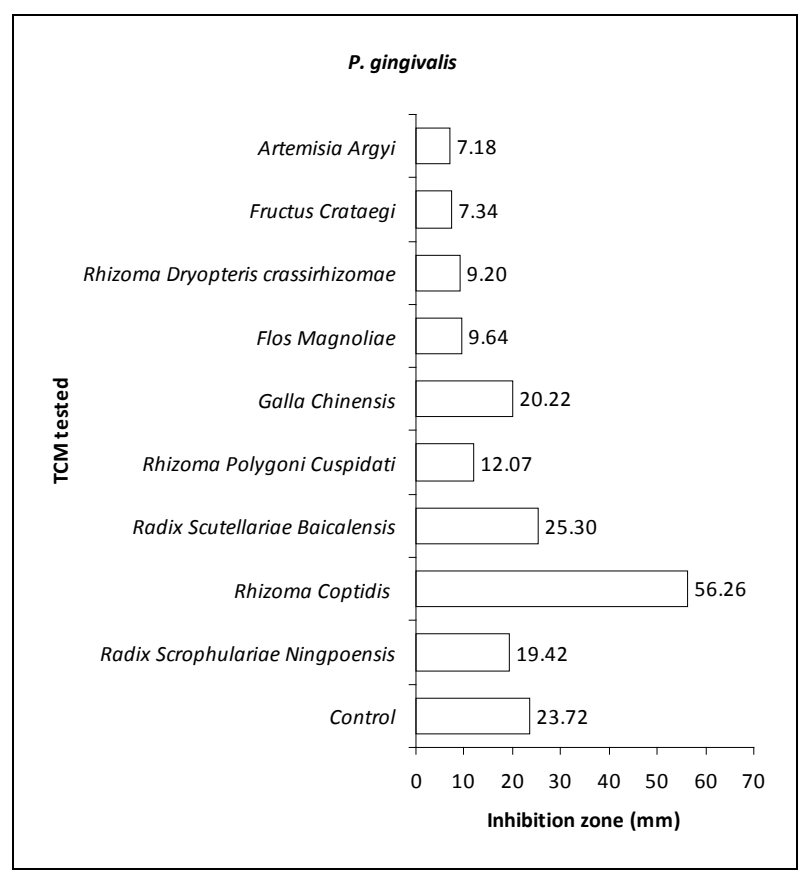

Figure 4. Sensitivity of $P$. gingivalis to 9 out of 20 TCM tested shown in mean value.

Table 2. MIC of Rhizoma copiditis and Galla chinensis against oral micro-organisms.

\begin{tabular}{lcc}
\hline \multicolumn{1}{c}{ Species } & $\begin{array}{c}\text { Rhizoma copiditis } \\
(\mathbf{g} / \mathbf{m L} \mathbf{)}\end{array}$ & $\begin{array}{c}\text { Galla chinensis } \\
\mathbf{( g / \mathbf { m L } )}\end{array}$ \\
\hline S. mutans & 0.039 & 0.039 \\
S. mitis & 0.020 & 0.010 \\
S. sanguis & 0.039 & 0.039 \\
P. gingivalis & $<0.001$ & $<0.001$ \\
\hline
\end{tabular}


Table 3. MBC of Rhizoma copiditis and Galla chinensis against oral micro-organisms.

\begin{tabular}{|c|c|c|}
\hline Species & $\begin{array}{c}\text { Rhizoma copiditis } \\
(\mathrm{g} / \mathrm{mL})\end{array}$ & $\begin{array}{l}\text { Galla chinensis } \\
(\mathrm{g} / \mathrm{mL})\end{array}$ \\
\hline S. mutans & 0.156 & 0.078 \\
\hline S. mitis & 0.156 & 0.078 \\
\hline S. sanguis & 0.039 & 0.039 \\
\hline P. gingivalis & $<0.001$ & $<0.001$ \\
\hline
\end{tabular}

Both MIC and MBC for Rhizoma Coptidis and Galla chinensis against $P$. gingivalis were below $<0.001 \mathrm{~g} / \mathrm{mL}$, which reached the lowest boundary for both MIC and MBC (Tables 2 and $\mathbf{3}$ ).

\section{Discussion}

In this study, twenty TCM extracts were evaluated for their antimicrobial activities against four common bacterial species presented in oral cavity which had been considered as important in biofilm formation (Streptococcus mitis, Streptococcus sanguis), or causing dental caries (Streptococcus mutans) or causing periodontal disease (Porphyromonas gingivalis).

Chlorhexidine was showed to be effective against all four tested bacteria. Of these, two TCM extracts, Rhizoma copiditis and Galla chinensis were shown to have similar effects. Although the first one has gained much attention due to its wide range of antimicrobial activities [5,7-9], ours is the first to show their effects specifically on oral bacteria. It has shown antimicrobial activity against periodontopathogenic bacteria including Porphyromonas gingivalis, Prevotella intermedia, Prevotella nigrescens and Actinobacillus actinomycetemcomitans Actinomyces naeslundii, but had less inhibitory effect on the growth of Streptococcus and Lactobacillus [5]. One active component, berberine alkaloid has been shown to have potent activity against all tested strains of methicillin-resistant Staphylococcus aureus (MRSA) [7]. Other studies demonstrated the antibacterial effect of Rhizoma copiditis and its alkaloids against Propionibacterium and Helicobacter pylori [8,9]. Results of the Control Group were published in a contemporary study evaluating another group of TCMs [10].

Results showed that both Rhizoma copiditis and Galla chinensis have comparable effects with chlorhexidine. When comparing the two TCMs, both Galla chinensis and Rhizoma Coptidis demonstrated the same MBC values against S. sanguis (Tables 2 and 3). Galla chinensis showed a lower MBC value against $S$. mitis and $S$. sanguis compared to Rhizoma Coptidis. This demonstrated that Galla chinensis has a higher bacteriocidal activity than Rhizoma Coptidis against the two bacteria. For P. gingivalis, both TCMs reached the lowest concentration boundary for the MIC and MBC values, further investigations are needed to determine the exact MIC and MBC values for both TCMs. This study is the first study that investigates TCMs specifically on oral bacteria. Therefore these TCMs are promising agents that can develop to new antibacterials for oral micro-organisms. Further research is needed to identify the specific active components that are related to the antibacterial action, to determine the range of action, and to investigate the mechanisms involved.

\section{Conclusions}

Both Rhizoma copitidis and Galla chinensis had inhibitory effects on Streptococcus mitis, Streptococcus sanguis, Streptococcus mutans and Porphyromonas gingivalis in vitro.

\section{Acknowledgements}

We thank Ms Joyce Yau on her technical assistance. This study was supported by the University Research Grant No.: 10207346.15633.08003.323.01, The University of Hong Kong.

\section{Conflict of Interest Statement}

The authors declare that there are no potential conflicts exist between the authors and the products mentioned in the paper.

\section{References}

[1] L. P. Samaranayake, "Essential Microbiology for dentistry,” Churchill Livingstone, Churchill, 2006.

[2] T. M. Auschill and N. Hein, "Effect of Two Antimicrobial Agents on early in Situ Biofilm Formation,” Journal of Clinical Periodontology, Vol. 32, No. 2, February 2005, pp. 147-152. doi:10.1111/j.1600-051X.2005.00650.x

[3] D. M. Eisenberg, "Trends in Alternative Medicine Use in the United States," Journal of the American Medical Association, Vol. 280, No. 18, 1998, pp. 1569-1575. doi:10.1001/jama.280.18.1569

[4] L. Wang, G. B. Zhou, P. Liu, J. H. Song, Y. Liang, X. J. Uan, F. Xu, B. S. Wang, J. H. Mao, Z. X. Shen, S. J. Chen and Z. Chen, "Dissection of Mechanisms of Chinese Medicinal Formula Realgar-Indigo Naturalis as an Effective Treatment for Promyelocytic Leukemic," Proceedings of the National Academy of Sciences, Vol. 105, No. 12, March 2008, pp. 4826-4831. doi:10.1073/pnas.0712365105

[5] J. P. Hu, N. Takahashi and T. Yamada, "Coptidis Rhizoma Inhibits Growth and Proteases of Oral Bacteria," Oral Disease, Vol. 6, No. 5, September 2000, pp. 297302. doi:10.1111/j.1601-0825.2000.tb00142.x

[6] M. Levison, “Antibacterial Therapy,” In: Vitro Testing, 
Pharmaco-Dynamics, Pharmacology, New Agents, Infectious Disease Clinics of North America, Vol. 9, 1995, pp. 483-495.

[7] H. H. Yu, K. J. Kim, J. D. Cha, H. K. Kim, Y. E. Lee, N. Y. Choi and Y. O. You, "Antimicrobial Activity of Berberine Alone and in Combination with Ampicillin or Oxacillin against Methicillin-Resistant Staphylococcus aureus," Journal of Medical Food, Vol. 8, No. 4, December 2005, pp. 454-461. doi:10.1089/jmf.2005.8.454

[8] E. A. Bae, M. J. Han, N. J. Kim and D. H. Kim, "AntiHelicobacter Pylori Activity of Herbal Medicines," Biological Pharmarcy Bulletin, Vol. 21, No. 9, Sep- tember 1998, pp. 990-992.

[9] S. Higaki, M. Nakamura, M. Morohashi, Y. Hasegawa and T. Yamagishi, "Anti-Lipase Activity of Kampo Formulations, Coptidis Rhizoma and Its Alkaloids against Propionibacterium Acnes,” Journal of Dermatology, Vol. 23, No. 5, 1996, pp. 310-314.

[10] R. W. K. Wong, U. Hagg, L Samaranayake, M. K. Z. Yuen, C. J. Seneviratne and R. Kao, “Antimicrobial Activity of Chinese Medicine Herbs against Common Bacteria in Oral Biofilm: A Pilot Study,” International Journal of Oral and Maxillofacial Surgery, Vol. 39, No. 6, 2010, pp. 599-605. doi:10.1016/j.ijom.2010.02.024 